\title{
Selection Combining of Signals with Different Modulation Levels in Nakagami-m Fading
}

\author{
Akram Bin Sediq and Halim Yanikomeroglu
}

\begin{abstract}
Conventionally, the BER analysis of selection combining schemes is performed under the assumption that the signals to be combined belong to the same modulation level. This is not necessarily the case in cooperative relaying, where the source and relay may use different modulation levels. Recently, a closed-form BER expression was derived for selection combing of signals with different modulation levels, under the assumption that the source-relay, source-destination, and relay-destination links are all modeled as Rayleigh fading channels. In this letter, we extend the analysis to the asymmetric Nakagami- $m$ fading channel, which is a more versatile channel model. We derive the closed-form BER expression which is expressed in terms of elementary functions. The derived BER expression generalizes many existing expressions in the literature. Simulation results are also presented to confirm the accuracy of the derived results.
\end{abstract}

Index Terms-Cooperative diversity, Nakagami- $m$ fading, BER selection combining, relay networks, diversity analysis.

\section{INTRODUCTION}

$\mathbf{I}$ $\mathrm{N}$ cooperative relaying, spatial diversity can be achieved by combining the signals from the source-relay and relaydestination links. Since the channel conditions in the sourcerelay and relay-destination links can be very different, the source (S) and relay (R) may use different modulation schemes. This introduces a new context for diversity combining, where the destination (D) combines signals with different modulation levels, in order to achieve spatial diversity.

In [1], BER performance analysis for combining signals with different modulation levels using soft-bit maximal ratio combining is presented. In [2], BER selection combining (BER-SC) was introduced, where receiver calculates the BER for each branch (using the SNR and the modulation level) and then decodes the signal from the branch that has the minimum BER. It is shown that BER-SC significantly outperforms conventional SNR selection combining (SNR-SC) where selection is based on SNRs only, when it is used for combining signals with different modulation levels. BER performance analysis was also presented for BER-SC and SNR$\mathrm{SC}$, assuming the channels in the S-R, S-D, and R-D links are all modeled as Rayleigh fading. In this letter, we build on our recent work published in [2], and extend the performance analysis to Nakagami- $m$ fading channels. The motivations of such an extension are two folds. First of all, the channel characteristic of the S-R, S-D, and R-D links are very different since the $S, R$, and $D$ can be far from each other which make them subject to different environments. Modeling these

Manuscript received January 10, 2012. The associate editor coordinating the review of this letter and approving it for publication was D. Michalopoulos.

A. Bin Sediq and H. Yanikomeroglu are with the Department of Systems and Computer Engineering, Carleton University, Ottawa, Canada (e-mail: \{akram, halim\}@sce.carleton.ca).

Digital Object Identifier 10.1109/LCOMM.2012.030512.120075 links as Nakagami- $m$ fading gives us the flexibility to model them more accurately. Secondly, the derived expressions for Nakagami- $m$ fading generalizes the expression provided in [2], since Rayleigh fading is a special case when $m=1$.

Notation: $\Gamma(m)$ is the gamma function defined by $\Gamma(m)=$ $\int_{0}^{\infty} t^{m-1} e^{-t} d t ; \Gamma(m, x)$ is the lower incomplete gamma function given by $\Gamma(m, x)=\int_{0}^{x} t^{m-1} e^{-t} d t$.

\section{SySTEM MODEL}

Similar to [2], we consider a three-node network, which consists of a source $(\mathrm{S})$, a relay $(\mathrm{R})$, and a destination (D), all having a single antenna. Half-duplexing relaying is considered, where $\mathrm{R}$ can either transmit or receive in a given time/frequency channel. As a result, $\mathrm{S}$ and $\mathrm{R}$ transmit on orthogonal channels. To facilitate discussion, time division duplexing is considered. S transmits an $N$-bits packet to both $\mathrm{R}$ and $\mathrm{D}$ in the first time slot, using $M_{S}$-QAM modulation. R decodes the packet, and checks whether it is received correctly using a cyclic redundancy check (CRC) code. If the packet is received correctly, $\mathrm{R}$ retransmits the packet using $M_{R}$-QAM modulation, and D use selection combining in order to decode the packet, i.e., $\mathrm{D}$ either decodes the packet from $\mathrm{S}$ or $\mathrm{R}$. If $\mathrm{R}$ can not decode, it remains silent and sends a one-bit negative acknowledgment to $\mathrm{D}$, and $\mathrm{D}$ decodes the packet from $\mathrm{S}$.

The main difference between the system model in this letter and the one considered in [2], is the use of Nakagami-m multipath fading channel model, instead of Rayleigh fading. In particular, the channel variations in the links S-R, S-D, and R-D are modeled as independent Nakagami- $m_{S R}$, Nakagami$m_{S D}$, and Nakagami- $m_{R D}$ random variables, respectively. We assume $m_{S R}, m_{S D}$, and $m_{R D}$ to be positive integers. The instantaneous SNRs (defined as the ratio between the received energy per symbol and the noise power spectral density) in the links S-R, S-D, and R-D are denoted by $\gamma_{S R}, \gamma_{S D}$, and $\gamma_{R D}$, respectively, and they are gamma-distributed random variables. The average SNRs in the links S-R, S-D, and R$\mathrm{D}$, are denoted by $\bar{\gamma}_{S R}, \bar{\gamma}_{S D}$, and $\bar{\gamma}_{R D}$, respectively. The distribution of $\gamma_{i}$, where $i \in\{S R, R D, S D\}$, is given by [3, p. 19]

$$
f_{m_{i}}\left(\gamma_{i}\right)=\frac{\left(m_{i} / \bar{\gamma}_{i}\right)^{m_{i}}}{\Gamma\left(m_{i}\right)} \gamma_{i}^{m_{i}-1} e^{-\frac{m_{i} \gamma_{i}}{\bar{\gamma}_{i}}} .
$$

\section{BER-BASED SELECTION COMBINING}

The selection criterion for BER-SC can be expressed as [2]

$$
\text { select node } i \text {, where } i=\arg \min _{i \in\{S, R\}} \operatorname{BER}_{M_{i}}\left(\gamma_{i D}\right) \text {. }
$$

For Gray coded square $M$-QAM, BER is well approximated by [4],

$$
\operatorname{BER}_{M_{i}}\left(\gamma_{i D}\right) \approx c_{M_{i}} Q\left(\sqrt{2 d_{M_{i}}^{2} \gamma_{i D}}\right),{ }^{1}
$$

\footnotetext{
${ }^{1}$ The expression is exact for $M_{i}=2$ (BPSK) and $M_{i}=4$ (QPSK).
} 
where

$$
\left(c_{M_{i}}, d_{M_{i}}\right)=\left\{\begin{array}{cl}
(1,1), & M_{i}=2, \\
\left(\frac{2-2 / \sqrt{M_{i}}}{\log _{2} \sqrt{M_{i}}}, \sqrt{\frac{3}{2\left(M_{i}-1\right)}}\right), & M_{i} \geq 4,
\end{array},\right.
$$

and $Q(x)$ is the tail probability of the standard normal distribution, given by $Q(x)=1 / \sqrt{2 \pi} \int_{x}^{\infty} e^{-t^{2} / 2} d t$.

It is shown in [2] that BER-SC is superior to SNRSC. Implementing BER-SC requires the evaluation of the $Q$ function. An approximate and simpler implementation is

$$
\left\{\begin{array}{ll}
\text { select node } \mathrm{S}, & \text { if } \gamma_{S D} \geq \rho \gamma_{R D} \\
\text { select node } \mathrm{R}, & \text { if } \gamma_{S D}<\rho \gamma_{R D}
\end{array},\right.
$$

where $\rho$ is a biasing factor, such that $\rho<1$ induces a bias towards selecting $\mathrm{S}$ and $\rho>1$ induces a bias towards selecting R. In [2], $\rho$ was given by $\rho=d_{M_{R}}^{2} / d_{M_{S}}^{2}$, in order to approximate BER-SC in the high SNR regime. This approximation is exact if $c_{M_{S}}=c_{M_{R}}$, and it is tight in the high SNR regime. Such an approximation doesn't only simplify the implementation but also simplifies the BER analysis, while providing performance very close to the implementation given by (2). We demonstrate later that this choice of $\rho$ leads to nearoptimal minimum average BER. Note that by setting $\rho=1$ (unbiased), we recover conventional SNR-SC.

Although we focus on square $M$-QAM modulations, all the derived equations are applicable to any modulation scheme that has instantaneous BER in the form $c_{M_{i}} Q\left(\sqrt{2 d_{M_{i}}^{2} \gamma_{i D}}\right)$.

\section{Performance Analysis in NaKagami- $m$ Fading}

In this section, we derive closed form expression for the average BER for arbitrary biasing factor $\rho$ and arbitrary positive integer Nakagami fading coefficients. The average BER can be written as [2]

$$
\operatorname{BER}^{(\rho)}=\operatorname{PER}_{S R} \operatorname{BER}_{S D}+\left(1-\operatorname{PER}_{S R}\right) \operatorname{BER}_{c o m p}^{(\rho)},
$$

where

$\mathrm{PER}_{S R}$ is the average packet error rate in the S-R link, $\mathrm{BER}_{S D}$ is the average BER in the S-D link, $\operatorname{BER}_{c o m p}^{(\rho)}$ is the average $\mathrm{BER}$ at $\mathrm{D}$ after selection combining with a biasing factor of $\rho$.

The derivations of $\operatorname{BER}_{c o m p}^{(\rho)}$ is given in Section IV-A and the derivations of $\mathrm{PER}_{S R}$ and $\mathrm{BER}_{S D}$ are given in Section IV-B. In Section IV-C, we give the final expression for $\mathrm{BER}^{(\rho)}$.

\section{A. Derivations of $\operatorname{BER}_{\text {comp }}^{(\rho)}$}

The instantaneous BER for the selection combining scheme given by (5), for any biasing factor $\rho$, can be expressed as

$$
\begin{aligned}
& \operatorname{BER}_{\text {comp }, \text { inst }}^{(\rho)} \approx \\
& \left\{\begin{array}{ll}
c_{M_{S}} Q\left(\sqrt{2 d_{M_{S}}^{2} \gamma_{S D}}\right), & \gamma_{S D} \geq \rho \gamma_{R D} \\
c_{M_{R}} Q\left(\sqrt{2 d_{M_{R}}^{2} \gamma_{R D}}\right), & \gamma_{S D}<\rho \gamma_{R D}
\end{array} .\right.
\end{aligned}
$$

A common approach to derive the average BER at the output of the combiner is to express the instantaneous BER as a function of the output SNR, and then average the instantaneous BER over the PDF of the output SNR (e.g. [3], [5]). However, it is not straight forward to use such an approach in the problem we are considering, where $M_{S} \neq M_{R}$, since in this case, the instantaneous BER (given by (7)) can not be expressed as a function of the output SNR only. As a result, we follow the framework introduced in [2], by averaging the instantaneous BER over the joint PDF of $\gamma_{S D}, \gamma_{R D}$, as follows:

$$
\begin{aligned}
\operatorname{BER}_{c o m p}^{(\rho)} \approx & \int_{0}^{\infty} \int_{\rho \gamma_{R D}}^{\infty} c_{M_{S}} Q\left(\sqrt{2 d_{M_{S}}^{2} \gamma_{S D}}\right) \\
& \times f_{m_{S D}}\left(\gamma_{S D}\right) f_{m_{R D}}\left(\gamma_{R D}\right) d \gamma_{S D} d \gamma_{R D} \\
& +\int_{0}^{\infty} \int_{\rho^{-1} \gamma_{S D}}^{\infty} c_{M_{R}} Q\left(\sqrt{2 d_{M_{R}}^{2} \gamma_{R D}}\right) \\
& \times f_{m_{S D}}\left(\gamma_{S D}\right) f_{m_{R D}}\left(\gamma_{R D}\right) d \gamma_{R D} d \gamma_{S D} .
\end{aligned}
$$

In order to evaluate the expression given by (8), we start by defining the following functions:

$$
\begin{aligned}
H_{m}(x ; b, c)= & \frac{(1 / c)^{m}}{\Gamma(m)} \int_{0}^{x} Q(\sqrt{2 b t}) t^{m-1} e^{-\frac{t}{c}} d t \\
= & \frac{1}{2}-\sum_{k=0}^{m-1} \frac{c^{-k}}{k !}\left(Q(\sqrt{2 b x}) x^{k} e^{-\frac{x}{c}}\right. \\
+ & \left.\sqrt{\frac{b}{4 \pi}}\left(\frac{1+b c}{c}\right)^{\left(-k-\frac{1}{2}\right)} \Gamma\left(k+\frac{1}{2},\left(\frac{1+b c}{c}\right) x\right)\right) \\
H_{m}(\infty ; b, c)= & \lim _{x \rightarrow \infty} H_{m}(x ; b, c) \\
= & \frac{1}{2}-\frac{1}{2} \sqrt{\frac{b c}{1+b c}} \sum_{k=0}^{m-1}\left(\begin{array}{c}
2 k \\
k
\end{array}\right) 4^{-k}(1+b c)^{-k} \\
J_{m, n}(x ; b, c, d)= & \frac{(1 / d)^{n}}{\Gamma(n)} \int_{0}^{\infty} H_{m}(x t ; b, c) t^{n-1} e^{-\frac{t}{d}} d t \\
= & \frac{1}{2}-\frac{1}{2}\left(\frac{\beta}{x d}\right)^{n} \sum_{k=0}^{m-1}\left(\begin{array}{c}
k+n-1 \\
k
\end{array}\right)\left(\frac{\beta}{c}\right)^{k} \\
& \times\left(1-\sqrt{\frac{b \beta}{1+b \beta}} \sum_{l=0}^{k+n-1}\left(\begin{array}{c}
2 l \\
l
\end{array}\right) 4^{-l}\right. \\
& \times(1+b \beta)^{-l}\left(1-\frac{l !}{(l-k) !} \frac{(n-1) !}{(k+n-1) !}\right. \\
& \left.\left.\times\left(\frac{\beta}{x d}\right)^{-k-n+l} 1_{l \geq k}\right)\right),
\end{aligned}
$$

where $\beta=\frac{c x d}{x d+c}$, and $1_{l \geq k}=1$ if $l \geq k$ and 0 otherwise. Details of deriving (9) and (11) are given in Appendix A and Appendix B, respectively. Using (11) and (10), $\mathrm{BER}_{\text {comp }}^{(\rho)}$ can be expressed as

$$
\begin{aligned}
\operatorname{BER}_{c o m p}^{(\rho)}= & c_{M_{S}} H_{m_{S D}}\left(\infty, d_{M_{S}}^{2}, \frac{\bar{\gamma}_{S D}}{m_{S D}}\right) \\
& -c_{M_{S}} J_{m_{S D}, m_{R D}}\left(\rho, d_{M_{S}}^{2}, \frac{\bar{\gamma}_{S D}}{m_{S D}}, \frac{\bar{\gamma}_{R D}}{m_{R D}}\right) \\
& +c_{M_{R}} H_{m_{R D}}\left(\infty, d_{M_{R}}^{2}, \frac{\bar{\gamma}_{R D}}{m_{R D}}\right) \\
& -c_{M_{R}} J_{m_{R D}, m_{S D}}\left(\rho^{-1}, d_{M_{R}}^{2}, \frac{\bar{\gamma}_{R D}}{m_{R D}}, \frac{\bar{\gamma}_{S D}}{m_{S D}}\right) .
\end{aligned}
$$

The expression given by (12) generalizes other expressions in the literature. For example, by setting $m_{S D}=m_{R D}=1$ (Rayleigh fading), and $\rho=1$ (SNR-SC) we get [2, Eq. 24]. Moreover, by setting $m_{S D}=m_{R D}=1$ and $\rho=d_{M_{R}}^{2} / d_{M_{S}}^{2}$ (BER-SC) we get [2, Eq. 20]. In addition, by setting $M_{S}=$ $M_{R}=2$, the expression in (12) gives a simplified version (in terms of elementary functions) of the BER expressions 


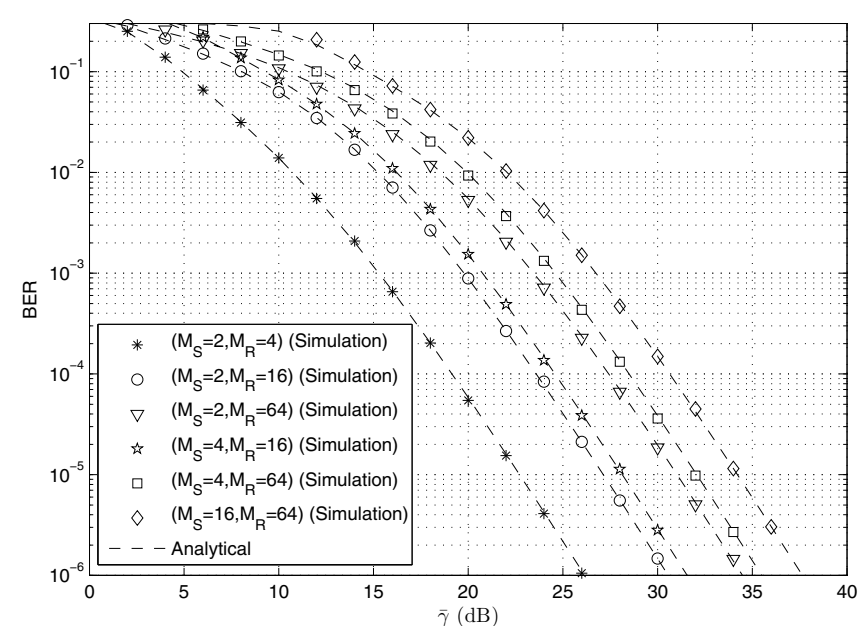

Fig. 1. BER performance of BER-SC for different modulation levels, assuming $m_{S R}=3, m_{S D}=1$, and $m_{R D}=2$.

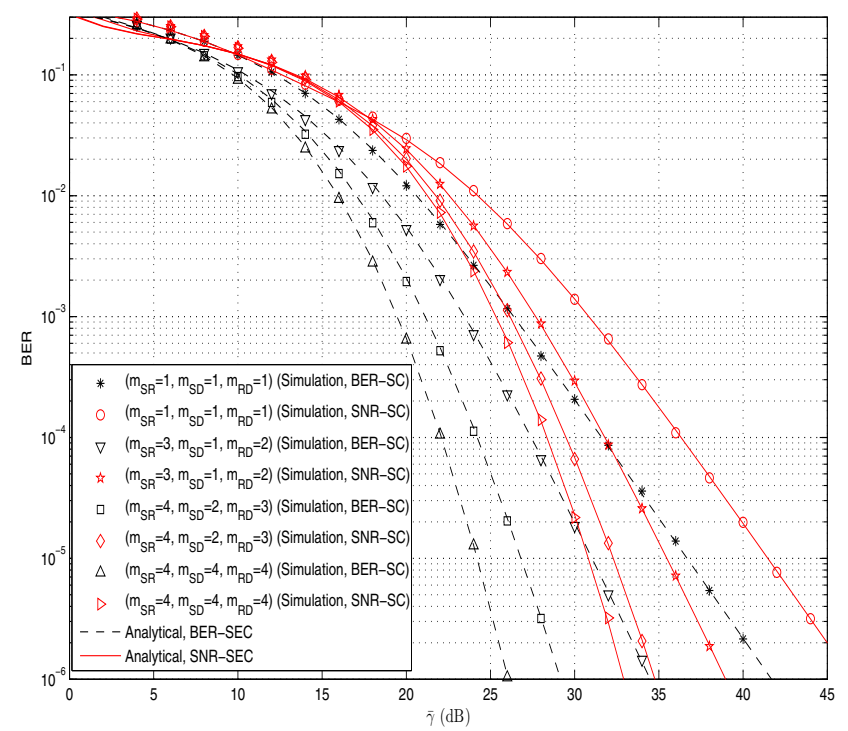

Fig. 2. BER performance of BER-SC and SNR-SC for different fading parameters, assuming $M_{S}=2$ and $M_{R}=64$.

given in [5, Eq. 26a] (in terms of Lauricella hypergeometric function), for the case of positive integer fading parameters.

In addition to cooperative relaying, the expression given by (12) can also be used in other applications where the same information is received in two orthogonal channels, using different modulation levels. For example, in two-transmissions Hybrid Automatic Repeat reQuest (HARQ), the channel may change in the second transmission, and it may be advantageous to use different modulation level in the second transmission. Also, in Coordinated Multi-Point (CoMP) transmission with orthogonal channels, the user terminal can be in an asymmetric position with respect to two different access points, and may receive the same information using different modulation levels.

\section{B. Derivations of $\mathrm{PER}_{S R}$ and $\mathrm{BER}_{S D}$}

The average BER in the link from node $i$ to $j$, where $i j \in$ $\{S R, R D, S D\}$, for $M$-QAM modulations in a point-to-point

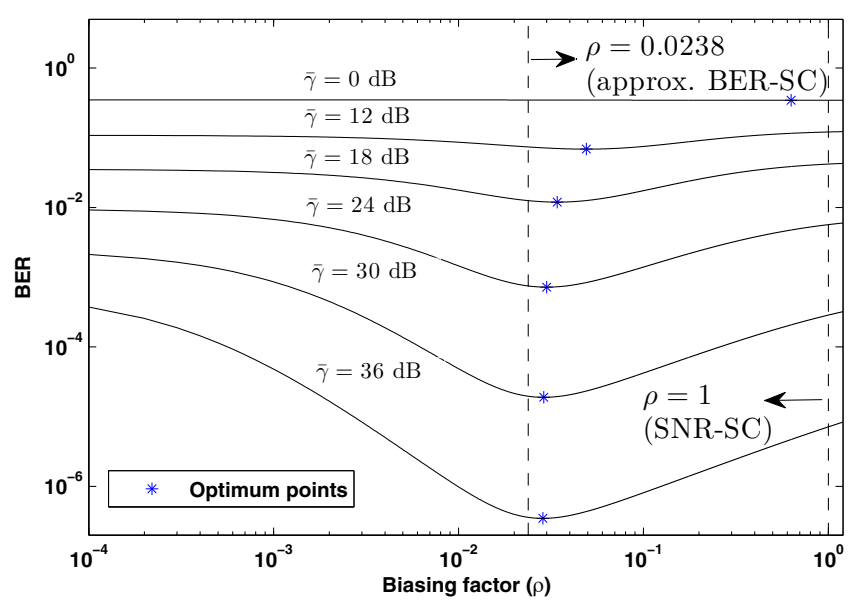

Fig. 3. BER versus biasing factor $(\rho)$, assuming $M_{S}=2, M_{R}=64$, $m_{S R}=3, m_{S D}=1$, and $m_{R D}=2$.

Nakagami- $m_{i j}$ fading channel can be well approximated as

$$
\begin{aligned}
\operatorname{BER}_{i j} & \approx \int_{0}^{\infty} c_{M_{i}} Q\left(\sqrt{2 d_{M_{i}}^{2} \gamma_{i j}}\right) f_{m i j}\left(\gamma_{i j}\right) d \gamma_{i j} . \\
& =c_{M_{i}} H_{m_{i j}}\left(\infty ; d_{M_{i}}^{2}, \frac{\bar{\gamma}_{i j}}{m_{i j}}\right)
\end{aligned}
$$

If symbol-errors occur independently in an $N$-bit packet, then $\mathrm{PER}_{S R}$ can be expressed as

$$
\begin{aligned}
\operatorname{PER}_{S R}= & 1-\left(1-\operatorname{SER}_{S R}\right)^{\frac{N}{\log _{2} M_{S}}} \\
\approx & 1-\left(1-\log _{2}\left(M_{S}\right) c_{M_{S}}\right. \\
& \left.\times H_{m_{S R}}\left(\infty ; d_{M_{S}}^{2}, \frac{\bar{\gamma}_{S R}}{m_{S R}}\right)\right)^{\frac{N}{\log _{2} M_{S}}},
\end{aligned}
$$

since SER $\approx \mathrm{BER} \log _{2} M_{S}$ for Gray-coded constellations [4].

\section{Final expressions for $\mathrm{BER}^{(\rho)}$}

By substituting (12), (14), and (13) in (6), the final expression for the average BER can be expressed as

$$
\begin{aligned}
& \operatorname{BER}^{(\rho)} \approx\left(1-\left(1-\log _{2}\left(M_{S}\right) c_{M_{S}}\right.\right. \\
& \left.\left.\times H_{m_{S R}}\left(\infty ; d_{M_{S}}^{2}, \frac{\bar{\gamma}_{S R}}{m_{S R}}\right)\right)^{\frac{N}{\log _{2} M_{S}}}\right) c_{M_{S}} H_{m_{S D}}\left(\infty ; d_{M_{S}}^{2}, \frac{\bar{\gamma}_{S D}}{m_{d}}\right) \\
& +\left(1-\log _{2}\left(M_{S}\right) c_{M_{S}} H_{m_{S R}}\left(\infty ; d_{M_{S}}^{2}, \frac{\bar{\gamma}_{S R}}{m_{S R}}\right)\right)^{\frac{N}{\log _{2} M_{S}}} \\
& \times\left(c_{M_{S}} H_{m_{S D}}\left(\infty, d_{M_{S}}^{2}, \frac{\bar{\gamma}_{S D}}{m_{S D}}\right)\right. \\
& -c_{M_{S}} J_{m_{S D}, m_{R D}}\left(\rho, d_{M_{S}}^{2}, \frac{\bar{\gamma}_{S D}}{m_{S D}}, \frac{\bar{\gamma}_{R D}}{m_{R D}}\right) \\
& +c_{M_{R}} H_{m_{R D}}\left(\infty, d_{M_{R}}^{2}, \frac{\bar{\gamma}_{R D}}{m_{R D}}\right) \\
& \left.-c_{M_{R}} J_{m_{R D}, m_{S D}}\left(\rho^{-1}, d_{M_{R}}^{2}, \frac{\bar{\gamma}_{R D}}{m_{R D}}, \frac{\bar{\gamma}_{S D}}{m_{S D}}\right)\right)
\end{aligned}
$$

where $H_{m}(\infty ; b, c)$ is given in (10) and $J_{m, n}(x ; b, c, d)$ is given in (11). We finally remark that the closed form expression given by (15) is expressed in terms of elementary functions and finite sums, which makes it easy to compute. 


\section{Analytical and Simulation Results}

We assume $\bar{\gamma}_{S R}=\bar{\gamma}+10 \mathrm{~dB}, \bar{\gamma}_{S D}=\bar{\gamma}-10 \mathrm{~dB}$, $\bar{\gamma}_{R D}=\bar{\gamma} \mathrm{dB}$, and $N=189$ bytes. In Fig. 1, we show the BER simulation results for BER-SC for different modulation levels. We also plot the analytical results for $\rho=d_{M_{R}}^{2} / d_{M_{S}}^{2}$. It is clear from the figure that the analytical results (given by (15)) are in excellent agreement with the simulation results. In Fig. 2, we show the simulation and analytical results for BER-SC and SNR-SC for different fading parameters. Again, there is an excellent agreement between the simulation and the analytical results for all parameters. Both BER-SC and SNR-SC achieve the same diversity order; however, BER$\mathrm{SC}$ achieves significantly higher SNR gain for all cases. For example, for the case of $m_{S R}=m_{R D}=m_{S D}=4$, the gain achieved by BER-SC over SNR-SC at BER $=10^{-3}$ is $5.9 \mathrm{~dB}$. In Fig. 3, we plot the BER given by (15) as a function of $\rho$, for different SNRs. For low SNRs, $\rho=d_{M_{R}}^{2} / d_{M_{S}}^{2}=0.0238$ is far from being optimum. However, the BER is not sensitive to the choice for $\rho$ for low SNRs. For high SNRs, this choice of $\rho$ gives near optimal BER and it yields much lower BER as compared to SNR-SC.

\section{CONCLUSIONS}

In this letter, we presented BER analysis for selection combining of signals with different modulation levels, in Nakagami- $m$ fading, where $m$ is a positive integer. The derived expression is applicable to asymmetric relay channels, where the S-R, S-D, and R-D links may have different fading coefficients and different average SNRs. The derived expression can be also used in other applications where the same information is received in two orthogonal channels, using different modulation levels, such as HARQ and CoMP. The derived formula is expressed in terms of elementary functions and it generalizes various expressions in the literature.

\section{APPENDIX A}

\section{DERIVATIONS OF $H_{m}(x ; b, c)$ GIVEN BY (9)}

To evaluate the integration given in (9), we start by using integration by parts by defining $U$ and $V$ as follows:

$$
\begin{aligned}
U= & \frac{(1 / c)^{m-1}}{\Gamma(m)} Q(\sqrt{2 b t}) t^{m-1} \rightarrow \\
d U= & \frac{(1 / c)^{m-1}}{\Gamma(m)}\left((m-1) Q(\sqrt{2 b t}) t^{m-2}\right. \\
& \left.-\sqrt{\frac{b}{4 \pi}} t^{m-\frac{3}{2}} e^{-b t}\right) d t \\
d V= & \frac{1}{c} e^{-\frac{t}{c}} \rightarrow V=-e^{-\frac{t}{c}} .
\end{aligned}
$$

Using (16), we can express the integration given in (9) as

$$
\begin{aligned}
H_{m}(x ; b, c) & =U V]_{0}^{x}-\int_{0}^{x} V d u \\
& =\left\{\begin{array}{ll}
h_{1}(x ; b, c)+\frac{1}{2}, & m=1 \\
h_{m}(x ; b, c)+H_{m-1}(x ; b, c), & m>1
\end{array},\right.
\end{aligned}
$$

where $h_{m}(x ; b, c)$ is given by

$$
\begin{aligned}
h_{m}(x ; b, c)= & -\frac{(1 / c)^{m-1}}{\Gamma(m)}\left(Q(\sqrt{2 b x}) x^{m-1} e^{-\frac{x}{c}}\right. \\
& \left.-\sqrt{\frac{b}{4 \pi}}\left(b+\frac{1}{c}\right)^{\left(-m+\frac{1}{2}\right)} \Gamma\left(m-\frac{1}{2},\left(b+\frac{1}{c}\right) x\right)\right) .
\end{aligned}
$$

By solving the recursive equation in (17), we get

$$
H_{m}(x ; b, c)=\sum_{k=0}^{m-1} h_{k+1}(x ; b, c) \text {. }
$$

By substituting (18) in (19), we get (9).

\section{APPENDIX B}

DERIVATIONS OF $J_{m, n}(x ; b, c, d)$ GIVEN BY (11)

$$
\begin{aligned}
& J_{m, n}(x ; b, c, d)=\frac{(1 / d)^{n}}{\Gamma(n)} \int_{0}^{\infty} H_{m}(x t ; b, c) t^{n-1} e^{-\frac{t}{d}} d t \\
& =\frac{1}{2}-\sum_{k=0}^{m-1} \frac{c^{-k}}{k !}\left((x d)^{k}\left(\frac{c}{x d+c}\right)^{k+n}\right. \\
& \left.\times \frac{\Gamma(k+n)}{\Gamma(n)} H_{k+n}\left(\infty, b x, \frac{c d}{x d+c}\right)+j_{n, k}(x ; b, c, d)\right) \\
& j_{n, k}(x ; b, c, d)=\sqrt{\frac{b}{4 \pi}}\left(\frac{c}{1+b c}\right)^{\left(k+\frac{1}{2}\right)} \frac{(1 / d)^{(n-1)}}{\Gamma(n)} \\
& \times \int_{0}^{\infty} \Gamma\left(k+\frac{1}{2},\left(\frac{1+b c}{c}\right) x t t^{n-1} \frac{1}{d} e^{-\frac{t}{d}} d t\right.
\end{aligned}
$$

To evaluate (21), we start by defining $U$ and $V$ as

$$
\begin{aligned}
U= & \Gamma\left(k+\frac{1}{2},\left(\frac{1+b c}{c}\right) x t\right) t^{n-1} \rightarrow \\
d U= & \left(t^{n-1} t^{k-\frac{1}{2}} e^{-\left(\frac{1+b c}{c}\right) x t}\left(\left(\frac{1+b c}{c}\right) x\right)^{k+\frac{1}{2}}\right. \\
& \left.+(n-1) t^{n-2} \Gamma\left(k+\frac{1}{2},\left(\frac{1+b c}{c}\right) x t\right)\right) d t \\
d V= & \frac{1}{d} e^{-\frac{t}{d}} d t \rightarrow V=-e^{-\frac{t}{d}} .
\end{aligned}
$$

Using (22) and integration by parts, we can express (21) as

$$
\begin{aligned}
j_{n, k}(x ; b, c, d)= & \sqrt{\frac{b}{4 \pi}}\left(\frac{c}{1+b c}\right)^{\left(k+\frac{1}{2}\right)} \frac{(1 / d)^{(n-1)}}{\Gamma(n)}(U V]_{0}^{\infty} \\
& \left.-\int_{0}^{\infty} V d U\right) \\
= & \sqrt{\frac{b}{4 \pi}} \frac{(1 / d)^{(n-1)}}{\Gamma(n)} x^{k+\frac{1}{2}}\left(\frac{x d+b c d x+c}{c d}\right)^{-n-k+\frac{1}{2}} \\
& \times \Gamma\left(n+k-\frac{1}{2}\right)+j_{n-1, k}(x ; b, c, d) .
\end{aligned}
$$

By solving the recursive equation in (23), we get

$$
\begin{aligned}
j_{n, k}(x ; b, c, d)= & \frac{1}{2}(x d)^{k} \sqrt{\frac{b c d x}{x d+b c d x+c}} \sum_{l=k}^{k+n-1}\left(\begin{array}{c}
2 l \\
l
\end{array}\right) \\
& \times \frac{l !}{(l-k) !} 4^{-l}\left(\frac{x d+b c d x+c}{c}\right)^{-l} .
\end{aligned}
$$

By substituting (24) and (10) in (20), and going through considerable simplifications, we get (11).

\section{REFERENCES}

[1] A. Bin Sediq and H. Yanikomeroglu, "Performance analysis of soft-bit maximal ratio combining in cooperative relay networks," IEEE Trans. Wireless Commun., vol. 8, no. 10, pp. 4934-3939, Oct. 2009.

[2] _ , "Performance analysis of selection combining of signals with different modulation levels in cooperative communications," IEEE Trans. Veh. Technol., vol. 60, no. 4, pp. 1880-1887, May 2011.

[3] M. Simon and M. Alouini, Digital Communication over Fading Channels: A Unified Approach to Performance Analysis. Wiley \& Sons, Inc., 2000.

[4] B. Sklar, Digital Communications: Fundamentals and Applications, 2nd edition. Prentice Hall, 2001.

[5] O. C. Ugweje, "Selection diversity for wireless communications in Nakagami-fading with arbitrary parameters," IEEE Trans. Veh. Technol., vol. 50, no. 6, pp. 1437-1448, Nov 2001. 